\title{
Untangling supernova-neutrino oscillations with beta-beam data
}

\author{
Natalie Jachowicz ${ }^{* \dagger}$ \\ Ghent University, Department of Subatomic and Radiation Physics, Proeftuinstraat 86, B-9000 \\ Gent, Belgium \\ E-mail: natalie.jachowicz@ugent.be
}

\section{G.C. McLaughlin}

Department of Physics, North Carolina State University, Raleigh, North Carolina 27695

E-mail: Gail_McLaughlin@ncsu.edu

\section{Volpe}

Institut de Physique Nucléaire, F-91406 Orsay cedex, France

E-mail: volpedipno.in2p3.fr

Neutrinos are the only particles able to reach the earth straight from the center of a core-collapse supernova. But the information that can be gathered from their signal in a terrestrial detector is limited by the fact that little experimental data on neutrino-nucleus interactions exists and by uncertainties in theoretical calculations. Neutrino oscillations are a further complication : the energy distribution of the neutrinos in a terrestrial detector will be different from the spectrum with which these neutrinos were first emitted. We show that low-energy beta-beams can provide ways to extract information about supernova-neutrinos and their oscillations in a model-independent way.

10th Symposium on Nuclei in the Cosmos

July 27 - August 12008

Mackinac Island, Michigan, USA

\section{*Speaker.}

${ }^{\dagger}$ N.J. thanks the Research Foundation Flanders (FWO) for financial support. G.C.M. acknowledges support from the Department of Energy, under contract DE-FG02-02ER41216. C.V. acknowledges the financial support of the EC under the FP6 'Research Infrastructure Action - Structuring the European Research Area' EURISOL DS Project, Contract Number 515768 RIDS. 


\section{Introduction}

Understanding the mechanism of a Type-II core-collapse supernova is a longstanding problem. While the general nature of these events is understood to be the explosion that results from the collapse of a massive star, a completely self-consistent model of the explosion does not yet exist. Weak interaction physics and neutrino-interactions are widely regarded as central players in the explosion mechanism. The core is so hot and dense that even the neutrinos are trapped in the collapse. Afterwards, these neutrinos scatter out of the core on a timescale of about ten seconds, taking away with them the vast majority of the gravitational binding energy released in the collapse.

Terrestrial neutrino telescopes can provide precious information about the processes going on in the center of the supernova. The information that can be inferred by the terrestrial detection of supernova neutrinos however suffers from the fact that the available data on neutrino-nucleus cross sections in this energy region is limited, due to the very small cross sections and due to the lack of monochromatic neutrino beams. Moreover the accuracy of theoretical predictions is influenced by uncertainties and model dependencies. The flexibility of the proposed beta-beam experiments [1,2], where neutrinos are produced in the decay of a primary beam of boosted ions can remedy some of these problems. The energy range interesting for supernova neutrino-physics can be covered by low-energy beta-beams [?, 4, 5].

In this contribution, we examine the use of a beta-beam experiment for the analysis of a supernova signal in a terrestrial detector. We demonstrate that beta-beam measurements can be of great help in the analysis of this signal, thereby avoiding the uncertainties involved in the theoretical modeling of neutrino-nucleus interactions and the limitations brought along by the fact that common neutrino beams are not mono-energetic. The central idea of the proposed technique consists of a reconstruction of supernova-neutrino responses using experimental beta-beam data, hence it is also of direct use for the prediction of neutrinonucleosynthesis reactions.

\section{Procedure}

Neutrinos and antineutrinos of different flavors decouple at different sites in the stellar core. In terms of energy, the supernova neutrinos are emitted with a low-energy and a high-energy component, the first associated with electron (anti)neutrinos, the latter with the heavy-flavor mu and tau neutrinos. Common parametrization are Fermi-Dirac or power-law spectra with average energies ranging from roughly 12 to $22 \mathrm{MeV}$, and varying width. Similar to supernova-neutrino spectra, beta-beam spectra are characterized by long tails, while their average energy and precise shape depend on the boost-factor $\gamma$ of the primary beam and the geometry of the experimental setup [6].

The core of the proposed technique consists of the construction of normalized linear combinations $n_{N \gamma}$ of beta-beam spectra $n_{\gamma_{i}}$ that then represent the supernova neutrino spectra:

$$
n_{N \gamma}\left(\varepsilon_{v}\right)=\sum_{i=1}^{N} a_{i} n_{\gamma_{i}}\left(\varepsilon_{v}\right)
$$

The boost factors $\gamma_{i=1, \cdots, N}$ and the expansion coefficients $a_{i=1, \cdots, N}$ are varied to minimize the expression

$$
\int_{\varepsilon_{v}} d \varepsilon_{v}\left|n_{N \gamma}\left(\varepsilon_{v}\right)-n_{S N}\left(\varepsilon_{v}\right)\right|
$$


In this way we obtain a synthetic spectrum $n_{N \gamma}^{f i t}\left(\varepsilon_{V}\right)$ that is the best fit to the supernova-neutrino energy-distribution $n_{S N}\left(\varepsilon_{v}\right)$ for particular values of the average energy and width.
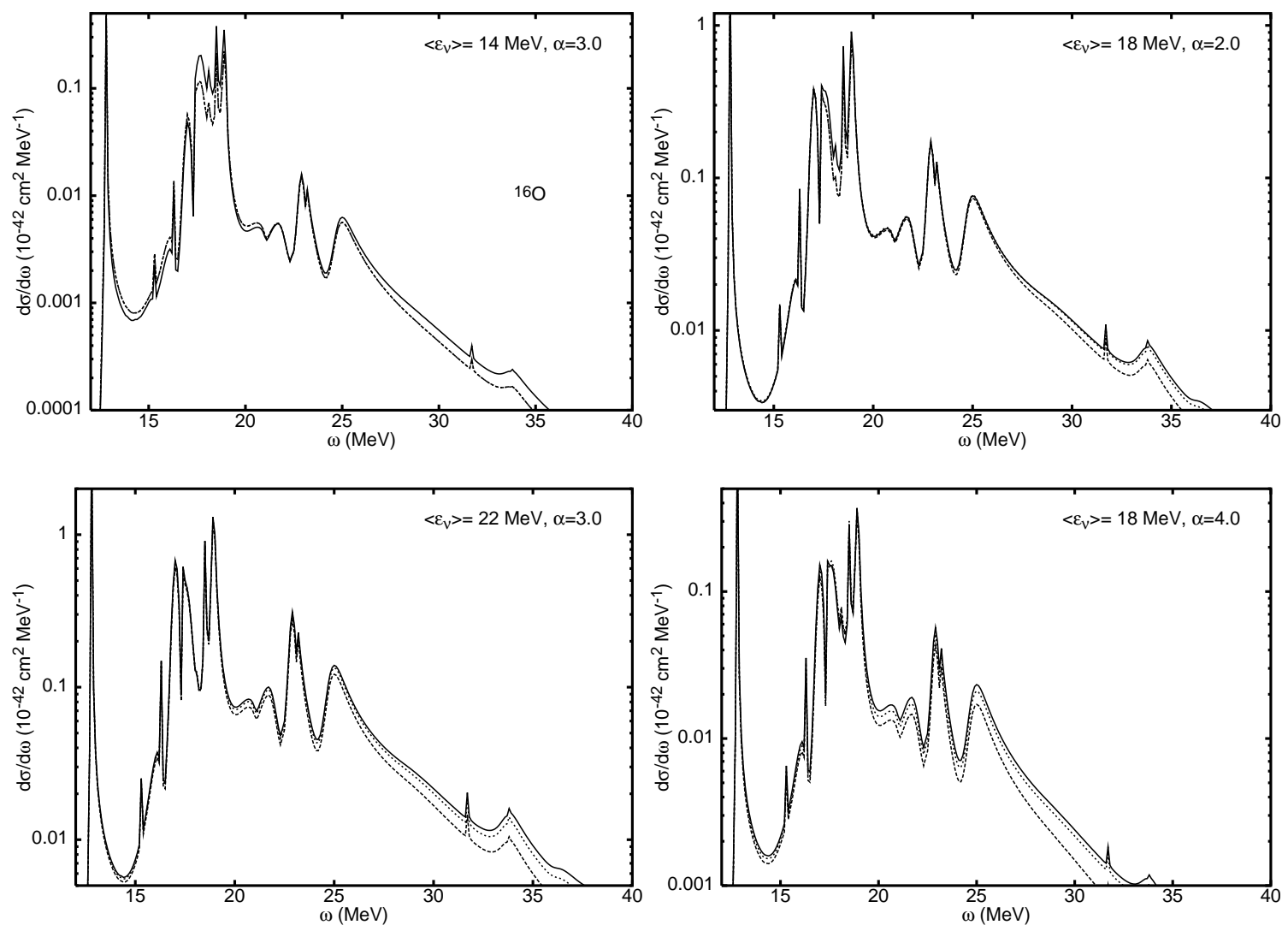

Figure 1: Comparison between differential cross sections for the reaction ${ }^{16} \mathrm{O}\left(v, v^{\prime}\right){ }^{16} \mathrm{O}^{*}$ folded with supernova neutrino spectra with different average energies and widths (full line), and with the corresponding synthetic spectra with $N=3$ (dashed) and $N=5$ (dotted) beta-beam spectra in the linear combination.

Figure 1 shows that the response produced by a synthetic spectrum i.e. the differential cross section folded with the linear combination of Eq.(2.1) is very similar to the supernova neutrino signal in a detector. Hence, using appropriate linear combinations of beta-beam data allows for very accurate predictions of supernova-neutrino responses without having to rely on the intermediate steps of nuclear structure calculations [7].

In fact, the accuracy of the fit is so satisfying that it opens ways to a model-independent study of supernova neutrino oscillations [8]. If we assume that neutrinos of all flavors are emitted in equal numbers, there will be twice as much high-energy neutrinos as low-energy neutrinos. In a terrestrial detector, both types will interact through neutral-current reactions. Charged-current detection channels are only accessible for electron-type neutrinos, as the production of a massive mu- or tau lepton requests more energy than is available for supernova neutrinos. Oscillations will transform the high and low-energy components in a different way. This does not affect the neutral current signal, as it is not sensitive to the neutrino flavor. In terms of the energy spectra, the neutral 

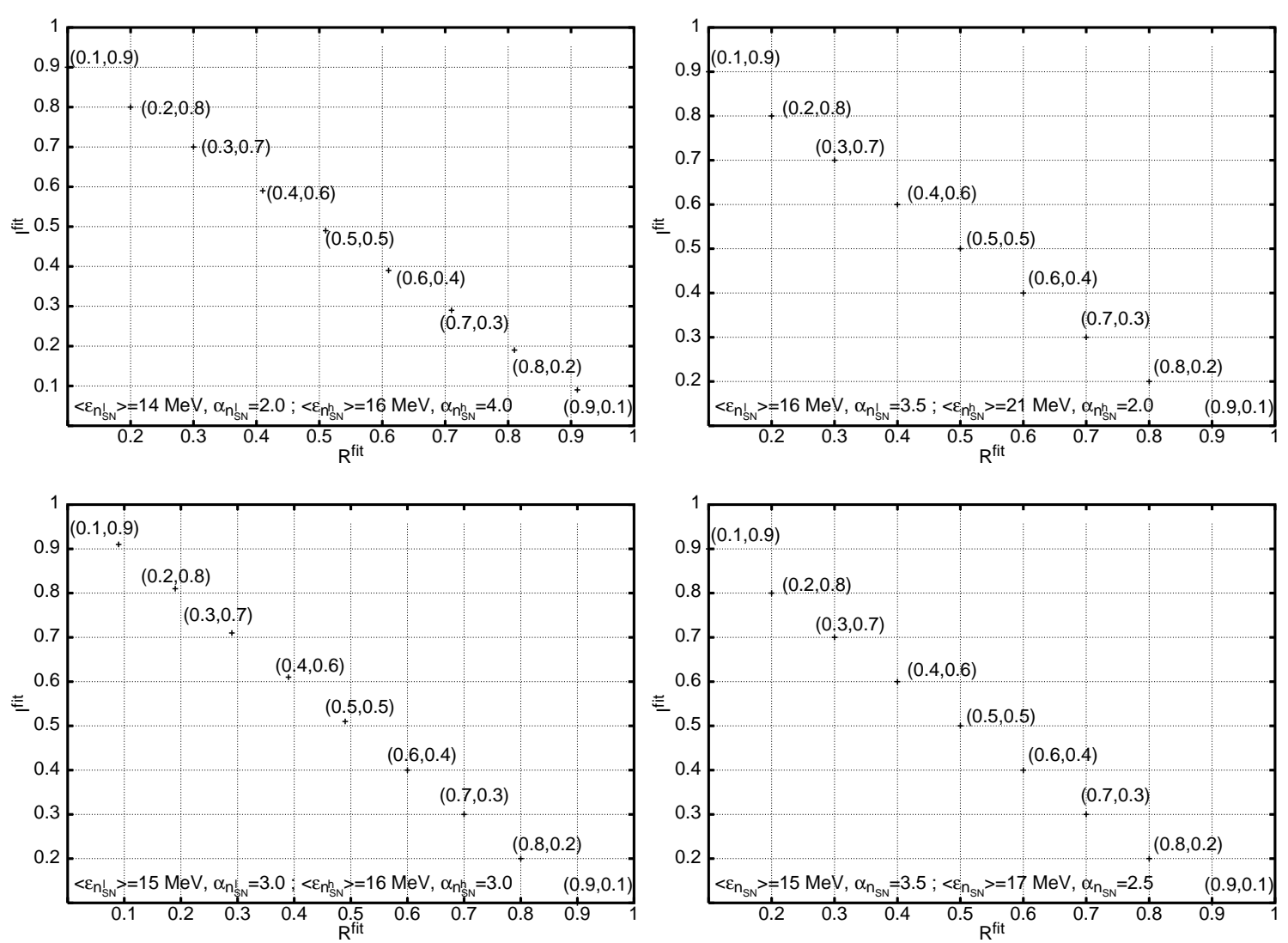

Figure 2: Extraction of the oscillation parameters $R^{\text {fit }}$ and $I^{\text {fit }}$ from a fit of the expressions in Eqs. (2.7) and (2.8) to the neutral $S_{N C}(\omega)=\int_{\varepsilon_{V}} d \varepsilon_{v}\left(n_{S N}^{l}\left(\varepsilon_{v}\right)+2 n_{S N}^{h}\left(\varepsilon_{v}\right)\right) \sigma_{N C}\left(\varepsilon_{v}, \omega\right)$, and charged-current $S_{C C}(\omega)=$ $\int_{\mathcal{E}_{v}} d \varepsilon_{v}\left(R n_{S N}^{l}\left(\varepsilon_{v}\right)+I n_{S N}^{h}\left(\varepsilon_{v}\right)\right) \sigma_{C C}\left(\varepsilon_{v}, \omega\right)$ supernova-neutrino signal in an oxygen detector. For each fitted point the original parameter values $(R, I)$ are given between brackets.

current signal can then be written as

$$
S_{N C}(\omega)=\int_{\varepsilon_{v}} d \varepsilon_{v}\left(n_{S N}^{l}\left(\varepsilon_{v_{e}}\right)+2 n_{S N}^{h}\left(\varepsilon_{v_{\mu}}, \varepsilon_{v_{\tau}}\right)+n_{S N}^{l}\left(\varepsilon_{\bar{v}_{e}}\right)+2 n_{S N}^{h}\left(\varepsilon_{\bar{v}_{\mu}}, \varepsilon_{\bar{v}_{\tau}}\right)\right) \sigma_{N C}\left(\varepsilon_{v}, \omega\right)
$$

where the energy integration runs over all neutrino flavors. The energy distribution of the neutrinos responsible for the charged current signal will be distorted according to :

$$
S_{C C}(\omega)=\int_{\varepsilon_{v}} d \varepsilon_{v}\left(R n_{S N}^{l}\left(\varepsilon_{v}\right)+I n_{S N}^{h}\left(\varepsilon_{v}\right)\right) \sigma_{C C}\left(\varepsilon_{v}, \omega\right)
$$

with $R$ denoting the fraction of electron-type neutrinos that remained unchanged and $I$ the fraction of heavy-flavor neutrinos that were transformed into electron neutrinos. In the equation above $R+$ $I=1$, for any given energy of the neutrinos. For simplicity, in our example below, we assume that the neutrinos will evolve either completely adiabatically or completely non-adiabatically so that in addition, both $R$ and $I$ are constant as a function of neutrino energy, although our analysis could be expanded to include the more general case. The parameters $R$ and $I$ contain the information about oscillations that the supernova neutrinos are carrying and can be recovered from the signal in the detector in two steps. First, from a set of constructed spectra $n_{N \gamma}$, the two linear combinations of 
beta-beam responses

$$
n_{N \gamma}^{l, f i t}\left(\varepsilon_{v}\right)=\sum_{i=1}^{N} a_{i}^{l, f i t} n_{\gamma_{i}}\left(\varepsilon_{v}\right)
$$

and

$$
n_{N \gamma}^{h, f i t}\left(\varepsilon_{v}\right)=\sum_{i=1}^{N} a_{i}^{h, f i t} n_{\gamma_{i}}\left(\varepsilon_{v}\right)
$$

for which

$$
2 \int_{\varepsilon_{v}} d \varepsilon_{v}\left(n^{l, f i t}\left(\varepsilon_{v}\right)+2 n^{h, f i t}\left(\varepsilon_{v}\right)\right) \sigma_{N C}\left(\varepsilon_{v}\right)
$$

minimizes the difference with the neutral current detector signal from Eq. (2.3) are selected. The resulting two sets of expansion parameters $\left(a_{i}^{f i t, l} ; i=1, N\right)$ and $\left(a_{i}^{f i t, h} ; i=1, N\right)$ are then used to construct combinations

$$
\int_{\mathcal{E}_{v}} d \varepsilon_{v}\left(R^{f i t} n^{l, f i t}\left(\varepsilon_{v}\right)+I^{f i t} n^{h, f i t}\left(\varepsilon_{v}\right)\right) \sigma_{C C}\left(\varepsilon_{v}\right)
$$

and seek for the oscillation parameters $R^{f i t}$ and $I^{f i t}$ that yield the best agreement between the expression of Eq. (2.8) and the signal of Eq. (2.4).

The results are presented in figure 2 , showing that the agreement between the original $(R, I)$ and the reconstructed parameters $\left(R^{f i t}, I^{\text {fit }}\right)$ is very good. Moreover, the reconstruction is stable against noise [8].

Concluding, we presented a method that may be of help in the analysis of a future supernovaneutrino signal. We propose to use the technique as a lever to make advantage of beta-beam data in untangling multiple neutrino spectra and disentangling the oscillation characteristics from mixed spectra. This is an essential feature of any model aiming at gaining information about the supernova mechanism, probing proto-neutron star physics, and understanding supernova nucleosynthesis, such as the neutrino process and the r-process. We have shown that the procedure is effective in extracting oscillation parameters from the signal in a terrestrial detector, illustrating the use of the technique for gathering information about the supernova as well as its neutrinos.

\section{References}

[1] P. Zucchelli, Phys. Lett. B 532, 166 (2002).

[2] http://beta-beam.web.cern.ch/beta-beam/.

[3] C. Volpe, J. Phys. G30, L1 (2004) [arXiv:hep-ph/0303222].

[4] P. S. Amanik and G. C. McLaughlin, Phys. Rev. C 75, 065502 (2007) [arXiv:hep-ph/0702207].

[5] C. Volpe, J. Phys. G34, R1 (2007) [arXiv:hep-ph/0605033].

[6] G. C. McLaughlin, Phys. Rev. C 70, 045804 (2004) [arXiv:nucl-th/0404002].

[7] N. Jachowicz and G. C. McLaughlin, Phys. Rev. Lett. 96, 172301 (2006) [arXiv:nucl-th/0604046].

[8] N. Jachowicz, G. C. McLaughlin, and C. Volpe, Phys. Rev. C 77, 055501 (2008). 